\title{
Determination of source term for the fractional Rayleigh-Stokes equation with random data
}

\author{
Tran Thanh Binh ${ }^{1}$, Dumitru Baleanu ${ }^{2,3,4}$, Nguyen Hoang Luc ${ }^{5}$ and Nguyen-H Can ${ }^{6 *}$ (D)
}

\author{
"Correspondence: \\ nguyenhuucan@tdtu.edu.vn \\ ${ }^{6}$ Applied Analysis Research Group, \\ Faculty of Mathematics and \\ Statistics, Ton Duc Thang University, \\ Ho Chi Minh City, Vietnam \\ Full list of author information is \\ available at the end of the article
}

\begin{abstract}
In this article, we consider the problem of finding a source term of a Rayleigh-Stokes equation. Our problem is not well-posed in the sense of Hadamard. The sought solution does not depend continuously on the given data. Using the truncation method and some new techniques on trigonometric estimators, we give the regularized solution. Moreover, the mean square error and convergence rates are established.
\end{abstract}

MSC: 35K05; 35K99; 47J06; 47H10

Keywords: Rayleigh-Stokes problem; Fractional derivative; III-posed problem; Random data

\section{Introduction}

In the future, there will be a large number of applications of fractional diffusion equations, especially in physics, environment, and some other areas [1-5]. Not only do these applications appear in fluid flow and heat conduction, but they are also appropriate to other topics in mathematics. There are many authors who have studied fractional partial differential equations and ordinary differential equations. Now, we can describe some interesting papers as follows. In [6], the authors considered a class of time-fractional reaction-diffusion equations with nonlocal boundary condition. In 2018, Yong et al. [7] studied Duhamel's formula for time-fractional Schrödinger equations. The existence and Hölder continuity of solutions for time-fractional Navier-Stokes equations have been studied by Zhou, Peng, and Huang [8]. In this paper, we study the following Rayleigh-Stokes problem:

$$
\begin{cases}\partial_{\mathbf{t}} u-\left(1+d \partial_{\mathbf{t}}^{\gamma}\right) \Delta u=\mathbf{F}(\mathbf{t}, \mathbf{x}), & (\mathbf{t}, \mathbf{x}) \in\left(0, \mathcal{T}_{0}\right) \times \Omega, \\ u(\mathbf{t}, \mathbf{x})=0, & \mathbf{x} \in \partial \Omega, \\ u(0, \mathbf{x})=0, & \mathbf{x} \in \Omega .\end{cases}
$$

Here, $\Omega \subset \mathbb{R}^{d}(d=1,2,3)$ is a smooth domain with the boundary $\partial \Omega$, and $\mathcal{T}_{0}>0$ is a given time. $u(\mathbf{t}, \mathbf{x})$ is the velocity and $d$ is constant with respect to $\mathbf{x}$ and $\mathbf{t}$, where $\mathbf{x}$ is the distance, $\mathbf{t}$ is the time, and $\partial_{\mathbf{t}}^{\gamma}$ denotes the Riemann-Liouville derivative of order $\gamma \in(0,1)[3,9]$.

(c) The Author(s) 2019. This article is licensed under a Creative Commons Attribution 4.0 International License, which permits use, sharing, adaptation, distribution and reproduction in any medium or format, as long as you give appropriate credit to the original author(s) and the source, provide a link to the Creative Commons licence, and indicate if changes were made.The images or other third party material in this article are included in the article's Creative Commons licence, unless indicated otherwise in a credit line to the material. If material is not included in the article's Creative Commons licence and your intended use is not permitted by statutory regulation or exceeds the permitted use, you will need to obtain permission directly from the copyright holder.To view a copy of this licence, visit http://creativecommons.org/licenses/by/4.0/. 
Our main goal is identifying the source term $F$ if we know the following final value data:

$$
u\left(\mathcal{T}_{0}, \mathbf{x}\right)=\mathbf{h}(\mathbf{x}), \quad \mathbf{x} \in \Omega,
$$

where the source function $F=\mathbf{F}(\mathbf{t}, \mathbf{x})=\mathcal{Q}(\mathbf{t}) f(\mathbf{x})$, and $\mathcal{Q}(\mathbf{t})$ is known in advance. It is clear that our problem as above is ill-posed by the meaning of Hadamard; in other words, there is not always the existence of a solution. In case the problem has a solution, then a small noise of an exact measurement can imply that the sought solution has large error. Hence, numerical computation is troublesome. So it is essential to have a regularization.

The Rayleigh-Stokes equation (1) is a principal part in the description of dynamic fluids [10]. More applications for such an equation can be found in [10,11]. The initial and boundary value problems for the Rayleigh-Stokes problem, called direct problems, have already been researched in [10]. In some previous papers, Dehghan et al. [12-15] considered some numeral solutions of the Rayleigh-Stokes problem. The initial value problem for the Rayleigh-Stokes equation has been studied by applying plenty of numeral methods such as the finite element method, etc. [10,16].

If the errors coming from unmanageable causes such as wind, rain, humidity, etc. appear, then the model will be considered to be random. The random situations can not directly use the approaches applying for deterministic cases. Sometimes, it is difficult to understand in calculating owing to the random noise. In some real cases, empirical measurements result in function $\mathbf{h}(\mathbf{x})$, which can be examined with many errors. When the function $\mathbf{h}(\mathbf{x})$ is measured at fixed points $\mathbf{x}_{\mathbf{k}} \in \Omega$, we can collect a set of $\mathbf{G}\left(\mathbf{x}_{\mathbf{k}}\right)$, where $\mathbf{G}\left(\mathbf{x}_{\mathbf{k}}\right) \approx \mathbf{h}\left(\mathbf{x}_{\mathbf{k}}\right)$. The errors regularly appear in observing practical measurements. Hence

$$
\mathbf{G}\left(\mathbf{x}_{\mathbf{k}}\right)=\mathbf{h}\left(\mathbf{x}_{\mathbf{k}}\right)+\varepsilon_{\mathbf{k}}, \quad \mathbf{k}=1, \ldots, \mathfrak{M},
$$

where $\varepsilon_{\mathbf{k}}, \mathbf{k}=1, \ldots, \mathfrak{M}$, are unknown independent random errors.

The points $\mathbf{x}_{\mathbf{k}}, \mathbf{k}=\overline{1, \mathfrak{M}}$, which are non-random, are called design points. We choose

$$
\mathbf{x}_{\mathbf{k}}=\frac{2 \mathbf{k}-1}{2 \mathfrak{M}}, \quad \mathbf{k}=1, \ldots, \mathfrak{M}
$$

and $\mathcal{D}=\left(\mathbf{G}\left(\mathbf{x}_{1}\right), \mathbf{G}\left(\mathbf{x}_{2}\right), \ldots, \mathbf{G}\left(\mathbf{x}_{\mathfrak{M}}\right)\right)$, which is the measure of

$$
\left(\mathbf{h}\left(\mathbf{x}_{1}\right), \mathbf{h}\left(\mathbf{x}_{2}\right), \ldots, \mathbf{h}\left(\mathbf{x}_{\mathfrak{M}}\right)\right) .
$$

Let us consider the random model as follows:

$$
\mathbf{G}\left(\mathbf{x}_{\mathbf{k}}\right)=\mathbf{h}\left(\mathbf{x}_{\mathbf{k}}\right)+\sigma_{\mathbf{k}} \varepsilon_{\mathbf{k}}
$$

Let us assume that $\varepsilon_{\mathbf{k}} \sim \mathcal{N}(0,1)$ is normally random variables, and the unknown errors $\sigma_{\mathbf{k}}, \mathbf{k}=1, \ldots, \mathfrak{M}$, are unknown independent noises. As we know, these unknown errors can come from many troubles as measuring environment or instrument, where $\sigma_{\mathbf{k}} \leq R_{\max }$, $\mathbf{k}=1, \ldots, \mathfrak{M}$, with $R_{\max }$ being the greatest possible error bound when measuring.

To determine the source value $f(\mathbf{x})$, we get the following assumptions: 
(a) $f(\mathbf{x}) \in L^{2}(\Omega)$ and $\mathbf{h}(\mathbf{x}) \in L^{2}(\Omega)$.

(b) $\mathbf{P}$ is a positive constant which is the a priori bound of function $f(\mathbf{x})$

$$
\|f\|_{\mathcal{H}^{\beta}(\Omega)} \leq \mathbf{P}, \quad \beta>0
$$

(c) The measured data $\mathcal{G}\left(x_{k}\right)$ and the function $\mathbf{h}(\mathbf{x})$ have a relation

$$
\mathcal{G}\left(\mathbf{x}_{\mathbf{k}}\right)=\mathbf{h}\left(\mathbf{x}_{\mathbf{k}}\right)+\sigma_{\mathbf{k}} \varepsilon_{\mathbf{k}}
$$

Now, we mention the aim and our methods of this paper. Section 2 and Sect. 3 provide the results to be used in the sequel. In Sect. 4, we obtain some estimations for regularity.

\section{Preliminaries}

First, we present the definitions of fractional operators and some notations.

Definition 2.1 For given function $f$, the following formula

$$
\partial_{\mathbf{t}}^{\gamma} f(\mathbf{t})=\frac{\partial}{\partial \mathbf{t}} \int_{0}^{\mathbf{t}} \omega_{1-\gamma}(\mathbf{t}-\mathfrak{s}) f(\mathfrak{s}) d \mathfrak{s}, \quad \omega_{\gamma}(\mathbf{t})=\frac{\mathbf{t}^{\gamma-1}}{\Gamma(\gamma)}
$$

is called the Riemann-Liouville integral of order $\gamma>0$. Here, $\Gamma(\cdot)$ stands for the gamma function.

Next we remind the $L^{2}(\Omega)$ space. The Neumann-Laplacian operator is defined by

$$
\mathcal{A} f(\mathbf{x}):=-\Delta f(\mathbf{x})=-\frac{\partial^{2} f(\mathbf{x})}{\partial \mathbf{x}^{2}} .
$$

By the spectral theory of the positive elliptic operator, the eigenvalues of $\mathcal{A}$ are $\lambda_{\mathfrak{n}}=\mathfrak{n}^{2}$. We denote the corresponding eigenfunctions by $\phi_{\mathfrak{n}}(\mathbf{x})=\sqrt{\frac{2}{\pi}} \sin (\mathfrak{n} \mathbf{x})$. Thus the eigenpairs $\left(\lambda_{\mathfrak{n}}, \phi_{\mathfrak{n}}\right), \mathfrak{n} \in \mathbb{Z}^{+}$, satisfy

$$
\begin{cases}\mathcal{A} \phi_{\mathfrak{n}}(\mathbf{x})=-\lambda_{\mathfrak{n}} \phi_{\mathfrak{n}}(\mathbf{x}), & \mathbf{x} \in \Omega, \\ \phi_{\mathfrak{n}}(\mathbf{x})=0, & \mathbf{x} \in \partial \Omega .\end{cases}
$$

The sequence $\left\{\phi_{\mathfrak{n}}\right\}_{\mathfrak{n} \in \mathbb{Z}^{+}}$is an orthonormal basis of $L^{2}(\Omega)$.

It admits the eigenvalues

$$
0 \leq \lambda_{1} \leq \lambda_{2} \leq \cdots \leq \lambda_{\mathfrak{n}} \leq \cdots
$$

with $\lambda_{\mathfrak{n}} \rightarrow \infty$ as $\mathfrak{n} \rightarrow \infty$ (see [12]). The corresponding eigenfunctions $\phi_{\mathfrak{n}} \in H_{0}^{1}(\Omega)$.

For any $p \geq 0$, denote the space

$$
\mathcal{H}^{p}(\Omega)=\left\{v \in L^{2}(\Omega) ; \sum_{n=1}^{\infty} \lambda_{\mathfrak{n}}^{p}\left|\left\langle v, \phi_{\mathfrak{n}}\right\rangle\right|^{2}<+\infty\right\} .
$$


We recall that $\mathcal{H}(\Omega)$ is a Banach space with the following norm:

$$
\|v\|_{\mathcal{H}^{p}(\Omega)}=\left(\sum_{\mathfrak{n}=1}^{\infty} \lambda_{\mathfrak{n}}^{p}\left|\left\langle v, \phi_{\mathfrak{n}}\right\rangle\right|^{2}\right)^{\frac{1}{2}}
$$

Now, we get the following lemmas.

Lemma 2.1 Let $\mathfrak{n}=1, \ldots, \mathfrak{M}-1$, with $\mathbf{x}_{\mathbf{k}}=\pi \frac{2 \mathbf{k}-1}{2 \mathfrak{M}}$ and $\phi_{\mathfrak{n}}\left(\mathbf{x}_{\mathbf{k}}\right)=\sqrt{\frac{2}{\pi}} \sin \left(\mathfrak{n} \mathbf{x}_{\mathbf{k}}\right)$, then for all $\mathfrak{m}=1,2, \ldots$, we have

$$
R_{\mathfrak{n}, \mathfrak{m}}=\sum_{\mathbf{k}=1}^{\mathfrak{M}-1} \phi_{\mathfrak{n}}\left(\mathbf{x}_{\mathbf{k}}\right) \phi_{\mathfrak{m}}\left(\mathbf{x}_{\mathbf{k}}\right)= \begin{cases}\frac{\mathfrak{M}}{\pi}, & \mathfrak{m} \pm \mathfrak{n}=2 l \mathfrak{M}(\text { l even }) \\ -\frac{\mathfrak{M}}{\pi}, & \mathfrak{m} \pm \mathfrak{n}=2 l \mathfrak{M}(\text { l odd }) \\ 0, & \text { otherwise }\end{cases}
$$

If $\mathfrak{m}=1, \ldots, \mathfrak{M}-1$, we obtain

$$
R_{\mathfrak{n}, \mathfrak{m}}= \begin{cases}\frac{\mathfrak{M}}{\pi}, & \mathfrak{m}=\mathfrak{n} \\ 0, & \mathfrak{m} \neq \mathfrak{n},\end{cases}
$$

and

$$
\sum_{i=1}^{\mathfrak{M}} \phi_{\mathfrak{n}}\left(\mathbf{x}_{k}\right)= \begin{cases}(-1)^{l} \mathfrak{M} \sqrt{\frac{2}{\pi}}, & \mathfrak{n}=2 l \mathfrak{M} \\ 0, & \mathfrak{n} \neq 2 l \mathfrak{M} .\end{cases}
$$

Lemma 2.2 Let $\mathfrak{n}, \mathfrak{M} \in \mathbb{Z}^{+}$such that $\mathfrak{n}=1, \ldots, \mathfrak{M}-1$. Assume that $\mathbf{h}$ is piecewise $C^{1}(\bar{\Omega})$ $\mathbf{x}_{\mathbf{k}}=\pi \frac{2 \mathbf{k}-1}{2 \mathfrak{M}}$ and $\phi_{\mathfrak{n}}\left(\mathbf{x}_{\mathbf{k}}\right)=\sqrt{\frac{2}{\pi}} \sin \left(\mathfrak{n} \mathbf{x}_{\mathbf{k}}\right)$, then

$$
\mathbf{h}_{\mathfrak{n}}=\frac{\pi}{\mathfrak{M}} \sum_{\mathbf{k}=1}^{\mathfrak{M}} \mathbf{h}\left(\mathbf{x}_{\mathbf{k}}\right) \phi_{\mathfrak{n}}\left(\mathbf{x}_{\mathbf{k}}\right)-\overline{\mathcal{G}}_{\mathfrak{n}, \mathfrak{M}}
$$

where

$$
\overline{\mathcal{G}}_{\mathfrak{n}, \mathfrak{M}}=\sum_{l=1}^{\infty}(-1)^{l}\left[\left\langle\mathbf{h}(\mathbf{x}), \phi_{\mathfrak{n}+2 l \mathfrak{M}}(\mathbf{x})\right\rangle+\left\langle\mathbf{h}(\mathbf{x}), \phi_{-\mathfrak{n}+2 l \mathfrak{M}}(\mathbf{x})\right\rangle\right] .
$$

Proof We will construct the discretization form of the Fourier coefficients. The function $\mathbf{h}$ can be written as follows:

$$
\mathbf{h}\left(\mathbf{x}_{\mathbf{k}}\right)=\sum_{\mathfrak{m}=1}^{\infty} \mathbf{h}_{\mathfrak{m}} \phi_{\mathfrak{m}}\left(\mathbf{x}_{\mathbf{k}}\right)
$$

where $\mathbf{h}_{\mathfrak{m}}=\left\langle\mathbf{h}(\mathbf{x}), \phi_{\mathfrak{m}}(\mathbf{x})\right\rangle$. This implies that

$$
\frac{1}{\mathfrak{M}} \sum_{\mathbf{k}=1}^{\mathfrak{M}} \mathbf{h}\left(\mathbf{x}_{\mathbf{k}}\right) \phi_{\mathfrak{n}}\left(\mathbf{x}_{\mathbf{k}}\right)=\frac{1}{\mathfrak{M}} \sum_{\mathbf{k}=1}^{\mathfrak{M}}\left[\sum_{\mathfrak{m}=1}^{\infty}\left\langle\mathbf{h}(\mathbf{x}), \phi_{\mathfrak{m}}(\mathbf{x})\right\rangle \phi_{\mathfrak{m}}(\mathbf{x})\right] \phi_{\mathfrak{n}}\left(\mathbf{x}_{\mathbf{k}}\right) .
$$


Using Lemma 2.1, we get

$$
\begin{aligned}
& \frac{1}{\mathfrak{M}} \sum_{\mathbf{k}=1}^{\mathfrak{M}} \mathbf{h}\left(\mathbf{x}_{\mathbf{k}}\right) \phi_{\mathfrak{n}}\left(\mathbf{x}_{\mathbf{k}}\right) \\
& \quad=\frac{1}{\mathfrak{M}} \sum_{\mathbf{k}=1}^{\mathfrak{M}}\left[\sum_{\mathfrak{m}=1}^{\mathfrak{M}} \mathbf{h}_{\mathfrak{m}} \phi_{\mathfrak{m}}\left(\mathbf{x}_{\mathbf{k}}\right)\right] \phi_{\mathfrak{n}}\left(\mathbf{x}_{\mathbf{k}}\right)+\frac{1}{\mathfrak{M}} \sum_{\mathbf{k}=1}^{\mathfrak{M}}\left[\sum_{\mathfrak{m}=\mathfrak{M}+1}^{\infty} \mathbf{h}_{\mathfrak{m}} \phi_{\mathfrak{m}}\left(\mathbf{x}_{\mathbf{k}}\right)\right] \phi_{\mathfrak{n}}\left(\mathbf{x}_{\mathbf{k}}\right) \\
& \quad=\frac{1}{\mathfrak{M}} \sum_{\mathbf{k}=1}^{\mathfrak{M}} \mathbf{h}_{\mathfrak{m}} \sum_{\mathfrak{m}=1}^{\mathfrak{M}} \phi_{\mathfrak{m}}\left(\mathbf{x}_{\mathbf{k}}\right) \phi_{\mathfrak{n}}\left(\mathbf{x}_{\mathbf{k}}\right)+\frac{1}{\mathfrak{M}} \sum_{\mathbf{k}=1}^{\mathfrak{M}} \mathbf{h}_{\mathfrak{m}} \sum_{\mathfrak{m}=\mathfrak{M}+1}^{\infty} \phi_{\mathfrak{m}}\left(\mathbf{x}_{\mathbf{k}}\right) \phi_{\mathfrak{n}}\left(\mathbf{x}_{\mathbf{k}}\right) \\
& \quad=\frac{1}{\pi} \mathbf{h}_{\mathfrak{n}}+\frac{1}{\pi} \sum_{l=1}^{\infty}(-1)^{l}\left[\left\langle\mathbf{h}(\mathbf{x}), \phi_{\mathfrak{n}+2 l \mathfrak{M}}(\mathbf{x})\right\rangle+\left\langle\mathbf{h}(\mathbf{x}), \phi_{-\mathfrak{n}+2 l \mathfrak{M}}(\mathbf{x})\right\rangle\right] \\
& \quad=\frac{1}{\pi} \mathbf{h}_{\mathfrak{n}}+\frac{1}{\pi} \overline{\mathcal{G}}_{\mathfrak{n}, \mathfrak{M}} .
\end{aligned}
$$

So the conclusion is completed.

\section{Mild solution of backward in time problem for Rayleigh-Stokes problem}

In this part, we establish a representation for the solution of problem (1). The solution $u$ is given by Fourier series as follows:

$$
u(\mathbf{t}, \mathbf{x})=\sum_{\mathfrak{n}=1}^{\infty}\left\langle u(\mathbf{t}, \mathbf{x}), \phi_{\mathfrak{n}}\right\rangle \phi_{\mathfrak{n}}(\mathbf{x})
$$

where

$$
\left\{\phi_{\mathfrak{n}}(\mathbf{x})=\sqrt{\frac{2}{\pi}} \sin (\mathfrak{n} \mathbf{x}),\left(\mathfrak{n} \in \mathbb{Z}^{+}\right)\right\}
$$

The reference [1] implies that

$$
\left\langle u(\mathbf{t}, \mathbf{x}), \phi_{\mathfrak{n}}(\mathbf{x})\right\rangle=\mathbf{S}_{\mathfrak{n}}(\mathbf{t}, \gamma)\left\langle u(0, \mathbf{x}), \phi_{\mathfrak{n}}(\mathbf{x})\right\rangle+\int_{0}^{\mathbf{t}} \mathbf{S}_{\mathfrak{n}}(\mathbf{t}-s, \gamma)\left\langle F(\mathfrak{s}, \cdot), \phi_{\mathfrak{n}}(\cdot)\right\rangle d \mathfrak{s},
$$

where $\mathbf{S}_{\mathfrak{n}}(\mathbf{t}, \gamma)$ is as follows:

$$
\mathcal{L}\left(\mathbf{S}_{\mathfrak{n}}(\mathbf{t}, \gamma)\right)=\frac{1}{\mathbf{t}+d \lambda_{\mathfrak{n}} \mathbf{t}^{\gamma}+\lambda_{\mathfrak{n}}}
$$

where $\mathcal{L}$ is the function with Laplace transform.

This implies that

$$
u(\mathbf{t}, \mathbf{x})=\sum_{\mathfrak{n}=1}^{\infty}\left[\mathbf{S}_{\mathfrak{n}}(\mathbf{t}, \gamma)\left\langle u(0, \mathbf{x}), \phi_{\mathfrak{n}}(\mathbf{x})\right\rangle+\int_{0}^{\mathbf{t}} \mathbf{S}_{\mathfrak{n}}(\mathbf{t}-\mathfrak{s}, \gamma)\left\langle F(\mathfrak{s}, \mathbf{x}), \phi_{\mathfrak{n}}(\mathbf{x})\right\rangle d \mathfrak{s}\right] \phi_{\mathfrak{n}}(\mathbf{x}) .
$$

Lemma 3.1 The function $\mathbf{S}_{\mathfrak{n}}(\mathbf{t}, \gamma), \mathfrak{n}=1,2, \ldots$, is equal to

$$
\mathbf{S}_{\mathfrak{n}}(\mathbf{t}, \gamma)=\int_{0}^{\infty} e^{-y \mathbf{t}} \mathcal{K}(\mathfrak{n}, y, \gamma) d y
$$


where

$$
\mathcal{K}(\mathfrak{n}, y, \gamma)=\frac{d}{\pi} \frac{\lambda_{\mathfrak{n}} \sin (\gamma \pi) y^{\gamma}}{\left(-y+\lambda_{\mathfrak{n}} d y^{\gamma} \cos (\gamma \pi)+\lambda_{\mathfrak{n}}\right)^{2}+\left(\lambda_{\mathfrak{n}} d y^{\gamma} \sin (\gamma \pi)\right)^{2}} .
$$

Proof See the proof in [17].

From Lemma 3.1, we derive some estimates.

Lemma 3.2 Assume that $\gamma \in(0,1)$. For all $\mathbf{t} \in\left[0, \mathcal{T}_{0}\right]$, the following estimates hold:

- There exists $\mathcal{B}_{1}\left(d, \mathcal{T}_{0}, \gamma\right)>0$ such that

$$
\mathbf{S}_{\mathfrak{n}}\left(\mathcal{T}_{0}, \gamma\right) \geq \frac{\mathcal{B}_{1}\left(d, \mathcal{T}_{0}, \gamma\right)}{\lambda_{\mathfrak{n}}}
$$

- There exists $\mathcal{B}_{2}(d, \gamma)>0$ such that

$$
\mathbf{S}_{\mathfrak{n}}(t, \gamma) \leq \frac{\mathcal{B}_{2}(d, \gamma)}{1+\lambda_{\mathfrak{n}} \mathbf{t}^{1-\gamma}}, \quad 0 \leq \mathbf{t} \leq \mathcal{T}_{0}
$$

where

$$
\mathcal{B}_{1}\left(d, \mathcal{T}_{0}, \gamma\right)=\frac{d \sin (\gamma \pi) e^{-\mathcal{T}_{0}}}{3 \pi(\gamma+1)\left(d^{2}+1+\frac{1}{\lambda_{1}^{2}}\right)}, \quad \mathcal{B}_{2}(d, \gamma)=\frac{\Gamma(1-\gamma)}{d \pi \sin (\gamma \pi)}+1
$$

Proof Using the inequality $\left(a_{1}+a_{2}+a_{3}\right)^{2} \leq 3\left(a_{1}^{2}+a_{2}^{2}+a_{3}^{2}\right)$ for any real numbers $a_{1}, a_{2}, a_{3}$, we obtain

$$
\left(-y+\lambda_{\mathfrak{n}} d y^{\gamma} \cos \gamma \pi+\lambda_{\mathfrak{n}}\right)^{2}+\left(\lambda_{\mathfrak{n}} d y^{\gamma} \sin \gamma \pi\right)^{2} \leq 3\left(\lambda_{\mathfrak{n}}^{2} d^{2} y^{2 \gamma}+\lambda_{\mathfrak{n}}^{2}+y^{2}\right)
$$

Hence

$$
\begin{aligned}
\mathbf{S}_{\mathfrak{n}}\left(\mathcal{T}_{0}, \gamma\right) & \geq \frac{d}{\pi} \sin (\gamma \pi) \int_{0}^{+\infty} \frac{e^{-y T_{0}} \lambda_{\mathfrak{n}} y^{\gamma} d y}{3\left(\lambda_{\mathfrak{n}}^{2} d^{2} y^{2 \gamma}+\lambda_{\mathfrak{n}}^{2}+y^{2}\right)} \\
& =\frac{d \sin (\gamma \pi)}{3 \pi \lambda_{\mathfrak{n}}} \int_{0}^{+\infty} \frac{e^{-y \mathcal{T}_{0}} y^{\gamma} d y}{d^{2} y^{2 \gamma}+1+\frac{y^{2}}{\lambda_{1}^{2}}} .
\end{aligned}
$$

Furthermore, we get

$$
\begin{aligned}
\int_{0}^{+\infty} \frac{e^{-y \mathcal{T}_{0}} y^{\gamma} d y}{d^{2} y^{2 \gamma}+1+\frac{y^{2}}{\lambda_{1}^{2}}} & \geq \int_{0}^{1} \frac{e^{-y \mathcal{T}_{0}} y^{\gamma} d y}{d^{2} y^{2 \gamma}+1+\frac{y^{2}}{\lambda_{1}^{2}}} \\
& \geq \frac{1}{d^{2}+1+\frac{1}{\lambda_{1}^{2}}} \int_{0}^{1} e^{-y \mathcal{T}_{0}} y^{\gamma} d y \\
& \geq \frac{e^{-\mathcal{T}_{0}}}{d^{2}+1+\frac{1}{\lambda_{1}^{2}}} \frac{1}{\gamma+1} .
\end{aligned}
$$


This gives that

$$
\begin{aligned}
\mathbf{S}_{\mathfrak{n}}\left(\mathcal{T}_{0}, \gamma\right) & \geq \frac{d \sin (\gamma \pi)}{3 \pi \lambda_{\mathfrak{n}}} \frac{e^{-\mathcal{T}_{0}}}{d^{2}+1+\frac{1}{\lambda_{1}^{2}}} \frac{1}{\gamma+1} \\
& \geq \frac{\mathcal{B}_{1}\left(d, \mathcal{T}_{0}, \gamma\right)}{\lambda_{\mathfrak{n}}}
\end{aligned}
$$

Note that

$$
\begin{aligned}
\mathcal{K}(\mathfrak{n}, y, \gamma) & =\frac{d}{\pi} \frac{\lambda_{\mathfrak{n}} \sin (\gamma \pi) y^{\gamma}}{\left(-y+\lambda_{\mathfrak{n}} d y^{\gamma} \cos (\gamma \pi)+\lambda_{\mathfrak{n}}\right)^{2}+\left(\lambda_{\mathfrak{n}} d y^{\gamma} \sin (\gamma \pi)\right)^{2}} \\
& \leq \frac{d}{\pi} \frac{\lambda_{\mathfrak{n}} \sin (\alpha \pi) y^{\alpha}}{\left(\lambda_{\mathfrak{n}} k y^{\alpha} \sin (\gamma \pi)\right)^{2}}=\frac{1}{k \pi \sin (\gamma \pi) \lambda_{\mathfrak{n}}} y^{-\gamma} .
\end{aligned}
$$

This implies that, for $\mathbf{t}>0$,

$$
\begin{aligned}
\mathbf{S}_{\mathfrak{n}}(\mathbf{t}, \gamma) & \leq \frac{1}{d \pi \sin (\gamma \pi) \lambda_{\mathfrak{n}}} \int_{0}^{\infty} e^{-\gamma \mathbf{t}} y^{-\gamma} d y \\
& =\frac{1}{d \pi \sin (\gamma \pi) \mathbf{t}^{1-\gamma} \lambda_{\mathfrak{n}}} \int_{0}^{\infty} e^{-\gamma \mathbf{t}}(y \mathbf{t})^{-\gamma} d(\gamma \mathbf{t}) \\
& \leq \frac{1}{d \pi \sin (\gamma \pi) \mathbf{t}^{1-\gamma} \lambda_{\mathfrak{n}}} \int_{0}^{\infty} e^{-\xi} \xi^{\gamma} d \xi=\frac{\Gamma(1-\gamma)}{d \pi \sin (\gamma \pi) \mathbf{t}^{1-\gamma} \lambda_{\mathfrak{n}}},
\end{aligned}
$$

where $\xi=y \mathbf{t}$, and we have the fact that

$$
\int_{0}^{\infty} e^{-\xi} \xi^{\gamma} d \xi=\Gamma(1-\gamma)
$$

Hence

$$
\mathbf{S}_{\mathfrak{n}}(\mathbf{t}, \gamma) \mathbf{t}^{1-\gamma} \lambda_{\mathfrak{n}} \leq \frac{\Gamma(1-\gamma)}{d \pi \sin (\gamma \pi)}
$$

Since $0<\mathbf{S}_{\mathfrak{n}}(\mathbf{t}, \gamma) \leq 1$ (see Theorem 2.2 , [17]), so we imply from that

$$
\mathbf{S}_{\mathfrak{n}}(\mathbf{t}, \gamma)\left(1+\mathbf{t}^{1-\gamma} \lambda_{\mathfrak{n}}\right) \leq \frac{\Gamma(1-\gamma)}{d \pi \sin (\gamma \pi)}+1
$$

Therefore

$$
\mathbf{S}_{\mathfrak{n}}(\mathbf{t}, \gamma) \leq \frac{\mathcal{B}_{2}(d, \gamma)}{1+\lambda_{\mathfrak{n}} \mathbf{t}^{1-\gamma}}, \quad 0 \leq \mathbf{t} \leq T
$$

Lemma 3.3 Let $\mathcal{Q}:\left[\mathcal{T}_{0}, 0\right] \rightarrow \mathbb{R}$ be a positive continuous function. Then we have, for all $\mathfrak{n} \in \mathbb{N}$

$$
\mathcal{Q}_{0} \frac{\mathcal{B}_{1}\left(d, \mathcal{T}_{0}, \gamma\right)}{\mathfrak{n}^{2}} \leq \int_{0}^{\mathcal{T}_{0}} \mathbf{S}_{\mathfrak{n}}(T-\mathfrak{s}, \gamma) \mathcal{Q}(\mathfrak{s}) d \mathfrak{s} \leq\|\mathcal{Q}\|_{\infty} \frac{\mathcal{B}_{2}(d, \gamma)}{\mathfrak{n}^{2}} \frac{\mathcal{T}_{0}^{\gamma}}{\gamma}
$$

where $\inf _{\mathbf{t} \in\left[0, \mathcal{T}_{0}\right]}|\mathcal{Q}(\mathbf{t})|=\mathcal{Q}_{0}>0$. Set $\|\mathcal{Q}\|_{\infty}=\sup _{\mathbf{t} \in\left[0, \mathcal{T}_{0}\right]}|\mathcal{Q}(\mathbf{t})|$. 
Proof As in the proof of Lemma 3.2, we obtain

$$
\begin{aligned}
\int_{0}^{\mathcal{T}_{0}} \mathbf{S}_{\mathfrak{n}}\left(\mathcal{T}_{0}-\mathfrak{s}, \gamma\right) d \mathfrak{s} & \leq \int_{0}^{\mathcal{T}_{0}} \frac{\mathcal{B}_{2}(d, \gamma)}{1+\lambda_{\mathfrak{n}}\left(\mathcal{T}_{0}-\mathfrak{s}\right)^{1-\gamma}} d \mathfrak{s} \\
& \leq \int_{0}^{\mathcal{T}_{0}} \frac{\left(\mathcal{T}_{0}-\mathfrak{s}\right)^{\gamma-1} \mathcal{B}_{2}(d, \gamma)}{\lambda_{\mathfrak{n}}} d \mathfrak{s} \\
& \leq \frac{\mathcal{B}_{2}(d, \gamma)}{\lambda_{\mathfrak{n}}} \int_{0}^{\mathcal{T}_{0}} \mathfrak{s}^{\gamma-1} d \mathfrak{s} \\
& \leq \frac{\mathcal{B}_{2}(d, \gamma)}{\lambda_{\mathfrak{n}}} \frac{\mathcal{T}_{0}^{\gamma}}{\gamma}
\end{aligned}
$$

This implies that

$$
\begin{aligned}
\int_{0}^{\mathcal{T}_{0}} \mathbf{S}_{\mathfrak{n}}\left(\mathcal{T}_{0}-\mathfrak{s}, \gamma\right) \mathcal{Q}(\mathfrak{s}) d \mathfrak{s} & \leq \sup _{\mathbf{t} \in\left[0, \mathcal{T}_{0}\right]}|\mathcal{Q}(\mathbf{t})| \int_{0}^{\mathcal{T}_{0}} \mathbf{S}_{\mathfrak{n}}\left(\mathcal{T}_{0}-\mathfrak{s}, \gamma\right) d \mathfrak{s} \\
& \leq\|\mathcal{Q}\|_{\infty} \frac{\mathcal{B}_{2}(d, \gamma)}{\lambda_{\mathfrak{n}}} \frac{\mathcal{T}_{0}^{\gamma}}{\gamma}
\end{aligned}
$$

and

$$
\begin{aligned}
\int_{0}^{\mathcal{T}_{0}} \mathbf{S}_{\mathfrak{n}}\left(\mathcal{T}_{0}-\mathfrak{s}, \gamma\right) \mathcal{Q}(\mathfrak{s}) d \mathfrak{s} & \geq \inf _{\mathbf{t} \in\left[0, \mathcal{T}_{0}\right]}|\mathcal{Q}(\mathbf{t})| \int_{0}^{\mathcal{T}_{0}} \mathbf{S}_{\mathfrak{n}}\left(\mathcal{T}_{0}-\mathfrak{s}, \gamma\right) d \mathfrak{s} \\
& \geq \mathcal{Q}_{0} \frac{\mathcal{B}_{1}\left(d, \mathcal{T}_{0}, \gamma\right)}{\lambda_{\mathfrak{n}}}
\end{aligned}
$$

The proof is completed.

\section{Main results}

The first result is given as follows.

Lemma 4.1 Assume $0<\mathcal{N}<\mathfrak{M}$, with $\mathcal{N} \in \mathbb{N}$. Let $h$ hold in Lemma 2.2. Under the conditions stated above, we have

$$
f(\mathbf{x})=\sum_{\mathfrak{n}=1}^{\mathcal{N}} \frac{\frac{\pi}{\mathfrak{M}} \sum_{\mathbf{k}=1}^{\mathfrak{M}} \mathbf{h}\left(\mathbf{x}_{\mathbf{k}}\right) \phi_{\mathfrak{n}}\left(\mathbf{x}_{\mathbf{k}}\right)-\overline{\mathcal{G}}_{\mathfrak{n}, \mathfrak{M}}}{\int_{0}^{\mathcal{T}_{0}} \mathbf{S}_{\mathfrak{n}}(\mathbf{t}-\mathfrak{s}, \gamma) \mathcal{Q}(\mathfrak{s}) d \mathfrak{s}} \phi_{\mathfrak{n}}(\mathbf{x})+\sum_{\mathfrak{n}=\mathcal{N}+1}^{\infty} \frac{\left\langle\mathbf{h}(\mathbf{x}), \phi_{\mathfrak{n}}(\mathbf{x})\right\rangle}{\int_{0}^{\mathcal{T}_{0}} \mathbf{S}_{\mathfrak{n}}(\mathbf{t}-\mathfrak{s}, \gamma) \mathcal{Q}(\mathfrak{s}) d \mathfrak{s}} \phi_{\mathfrak{n}}(\mathbf{x})
$$

Proof First, we have the following equality.

Substituting $\mathbf{t}$ by $\mathcal{T}_{0}$ into equation (12), by the supplementary condition $u\left(\mathcal{T}_{0}, \mathbf{x}\right)=\mathbf{h}(\mathbf{x})$ and $u(0, \mathbf{x})=0$, we have

$$
\left\langle\mathbf{h}(\mathbf{x}), \phi_{\mathfrak{n}}(\mathbf{x})\right\rangle=\left\langle f(\mathbf{x}), \phi_{\mathfrak{n}}(\mathbf{x})\right\rangle \int_{0}^{\mathcal{T}_{0}} \mathbf{S}_{\mathfrak{n}}\left(\mathcal{T}_{0}-\mathfrak{s}, \gamma\right) \mathcal{Q}(\mathfrak{s}) d \mathfrak{s}
$$

where $\left\langle F(\mathfrak{s}, \mathbf{x}), \phi_{\mathfrak{n}}(\mathbf{x})\right\rangle=\left\langle\mathcal{Q}(\mathfrak{s}) f(\mathbf{x}), \phi_{\mathfrak{n}}(\mathbf{x})\right\rangle=\mathcal{Q}(\mathfrak{s})\left\langle f(\mathbf{x}), \phi_{\mathfrak{n}}(\mathbf{x})\right\rangle$.

The source function $f$ is given by

$$
f(\mathbf{x})=\sum_{\mathfrak{n}=1}^{\infty}\left\langle f(\mathbf{x}), \phi_{\mathfrak{n}}(\mathbf{x})\right\rangle=\sum_{\mathfrak{n}=1}^{\infty} \frac{\left\langle\mathbf{h}(\mathbf{x}), \phi_{\mathfrak{n}}(\mathbf{x})\right\rangle}{\int_{0}^{\mathcal{T}_{0}} \mathbf{S}_{\mathfrak{n}}\left(\mathcal{T}_{0}-\mathfrak{s}, \gamma\right) \mathcal{Q}(\mathfrak{s}) d \mathfrak{s}} \phi_{\mathfrak{n}}(\mathbf{x})
$$


Using Lemma 3.2, we have

$$
\begin{aligned}
\sum_{\mathfrak{n}=1}^{\infty} & \frac{\left\langle\mathbf{h}(\mathbf{x}), \phi_{\mathfrak{n}}(\mathbf{x})\right\rangle \phi_{\mathfrak{n}}(\mathbf{x})}{\int_{0}^{\mathcal{T}_{0}} \mathbf{S}_{\mathfrak{n}}\left(\mathcal{T}_{0}-\mathfrak{s}, \gamma\right) \mathcal{Q}(\mathfrak{s}) d \mathfrak{s}} \\
= & \sum_{\mathfrak{n}=1}^{\mathcal{N}} \frac{\left\langle\mathbf{h}(\mathbf{x}), \phi_{\mathfrak{n}}(\mathbf{x})\right\rangle \phi_{\mathfrak{n}}(\mathbf{x})}{\int_{0}^{\mathcal{T}_{0}} \mathbf{S}_{\mathfrak{n}}\left(\mathcal{T}_{0}-\mathfrak{s}, \gamma\right) \mathcal{Q}(\mathfrak{s}) d \mathfrak{s}}+\sum_{\mathfrak{n}=\mathcal{N}+1}^{\infty} \frac{\left\langle\mathbf{h}(\mathbf{x}), \phi_{\mathfrak{n}}(\mathbf{x})\right\rangle \phi_{\mathfrak{n}}(\mathbf{x})}{\int_{0}^{\mathcal{T}_{0}} \mathbf{S}_{\mathfrak{n}}\left(\mathcal{T}_{0}-\mathfrak{s}, \gamma\right) \mathcal{Q}(\mathfrak{s}) d \mathfrak{s}} \\
= & \sum_{\mathfrak{n}=1}^{\mathcal{N}} \frac{\left(\frac{\pi}{\mathfrak{M}} \sum_{\mathbf{k}=1}^{\mathfrak{M}} \mathbf{h}\left(\mathbf{x}_{\mathbf{k}}\right) \phi_{\mathfrak{n}}\left(\mathbf{x}_{\mathbf{k}}\right)-\overline{\mathcal{G}}_{\mathfrak{n}, \mathfrak{M}}\right) \phi_{\mathfrak{n}}(\mathbf{x})}{\int_{0}^{\mathcal{T}_{0}} \mathbf{S}_{\mathfrak{n}}(T-\mathfrak{s}, \gamma) \mathcal{Q}(\mathfrak{s}) d \mathfrak{s}} \\
& +\sum_{\mathfrak{n}=\mathcal{N}+1}^{\infty} \frac{\left\langle\mathbf{h}(\mathbf{x}), \phi_{\mathfrak{n}}(\mathbf{x})\right\rangle \phi_{\mathfrak{n}}(\mathbf{x})}{\int_{0}^{\mathcal{T}_{0}} \mathbf{S}_{\mathfrak{n}}\left(\mathcal{T}_{0}-\mathfrak{s}, \gamma\right) \mathcal{Q}(\mathfrak{s}) d \mathfrak{s}}
\end{aligned}
$$

The proof is completed.

\subsection{The ill-posedness of the problem}

In order to illustrate the ill-posedness of the backward problem through an example, let $h=0$ and $h\left(\mathbf{x}_{\mathbf{k}}\right)=0$. This implies $f=0$, and define function $u_{\mathfrak{M}}\left(\mathcal{T}_{0}, \mathbf{x}_{\mathbf{k}}\right)=\frac{1}{\sqrt{\mathfrak{M}}} \varepsilon_{\mathbf{k}}$. First, we have

$$
u_{\mathfrak{M}}\left(\mathcal{T}_{0}, \mathbf{x}\right)=\sum_{\mathfrak{n}=1}^{\mathfrak{M}-1}\left(\frac{\pi}{\mathfrak{M}} \sum_{\mathbf{k}=1}^{\mathfrak{M}} u_{\mathfrak{M}}\left(\mathcal{T}_{0}, \mathbf{x}_{\mathbf{k}}\right) \phi_{\mathfrak{n}}\left(\mathbf{x}_{\mathbf{k}}\right)\right) \phi_{\mathfrak{n}}(\mathbf{x})
$$

Let $f_{\mathfrak{M}}$ be the source function of the problem

$$
\left\{\begin{array}{l}
\partial_{\mathbf{t}} u_{\mathfrak{M}}-\left(1+d \partial_{\mathbf{t}}^{\gamma}\right) \Delta u_{\mathfrak{M}}=\mathcal{Q}(\mathbf{t}) f_{\mathfrak{M}}(\mathbf{x}) \\
u_{\mathfrak{M}}(\mathbf{t}, \mathbf{x})=0 \\
u_{\mathfrak{M}}(0, \mathbf{x})=0 \\
u_{\mathfrak{M}}\left(\mathcal{T}_{0}, \mathbf{x}\right)=\sum_{\mathfrak{n}=1}^{\mathfrak{M}-1}\left(\frac{\pi}{\mathfrak{M}} \sum_{\mathbf{k}=1}^{\mathfrak{M}} u_{\mathfrak{M}}\left(\mathcal{T}_{0}, \mathbf{x}_{\mathbf{k}}\right) \phi_{\mathfrak{n}}\left(\mathbf{x}_{\mathbf{k}}\right)\right) \phi_{\mathfrak{n}}(\mathbf{x})
\end{array}\right.
$$

Moreover, by the Parseval equality, we have

$$
\left\|u_{\mathfrak{M}}\right\|_{L^{2}(\Omega)}^{2}=\sum_{\mathfrak{n}=1}^{\mathfrak{M}-1}\left(\frac{\pi}{\mathfrak{M}} \sum_{\mathbf{k}=1}^{\mathfrak{M}} u_{\mathfrak{M}}\left(\mathcal{T}_{0}, \mathbf{x}_{\mathbf{k}}\right) \phi_{\mathfrak{n}}\left(\mathbf{x}_{\mathbf{k}}\right)\right)^{2}
$$

Using $\mathbb{E}\left(\varepsilon_{j} \varepsilon_{l}\right)=0(j \neq l), \mathbb{E}\left(\varepsilon_{j}\right)=0(j=1,2, \ldots, \mathfrak{n})$, and Lemma 2.1 , we can deduce that

$$
\begin{aligned}
\mathbb{E}\left\|u_{\mathfrak{M}}\right\|_{L^{2}(\Omega)}^{2} & =\sum_{\mathfrak{n}=1}^{\mathfrak{M}-1}\left(\frac{\pi}{\mathfrak{M}} \sum_{\mathbf{k}=1}^{\mathfrak{M}} \frac{1}{\sqrt{\mathfrak{M}}} \mathbb{E} \varepsilon_{\mathbf{k}} \phi_{\mathfrak{n}}\left(\mathbf{x}_{\mathbf{k}}\right)\right)^{2} \\
& =\sum_{\mathfrak{n}=1}^{\mathfrak{M}-1}\left(\frac{\pi^{2}}{\mathfrak{M}^{2}} \sum_{\mathbf{k}=1}^{\mathfrak{M}} \frac{1}{\sqrt{\mathfrak{M}}} \phi_{\mathfrak{n}}\left(\mathbf{x}_{\mathbf{k}}\right)\right)^{2}=\frac{\pi}{\mathfrak{M}} .
\end{aligned}
$$

Then

$$
\lim _{\mathfrak{M} \rightarrow \infty} \mathbb{E}\left\|u_{\mathfrak{M}}\right\|_{L^{2}(\Omega)}^{2}=0
$$


We have

$$
\begin{aligned}
f_{\mathfrak{M}}(x)= & \sum_{\mathfrak{n}=1}^{\mathfrak{M}-1} \frac{\frac{\pi}{\mathfrak{M}} \sum_{\mathfrak{k}=1}^{\mathfrak{M}} u_{\mathfrak{M}}\left(\mathcal{T}_{0}, \mathbf{x}_{\mathbf{k}}\right) \phi_{\mathfrak{n}}\left(\mathbf{x}_{\mathbf{k}}\right)-\widetilde{\mathcal{G}}_{\mathfrak{n}, \mathfrak{M}}}{\int_{0}^{\mathcal{T}_{0}} \mathbf{S}_{\mathfrak{n}}(\mathbf{t}-\mathfrak{s}, \gamma) \mathcal{Q}(\mathfrak{s}) d \mathfrak{s}} \phi_{\mathfrak{n}}(\mathbf{x}) \\
& +\sum_{\mathfrak{n}=\mathfrak{M}+1}^{\infty} \frac{\left\langle u_{\mathfrak{M}}\left(\mathcal{T}_{0}, \mathbf{x}\right), \phi_{\mathfrak{n}}(\mathbf{x})\right\rangle}{\int_{0}^{\mathcal{T}_{0}} \mathbf{S}_{\mathfrak{n}}(\mathbf{t}-\mathfrak{s}, \gamma) \mathcal{Q}(\mathfrak{s}) d \mathfrak{s}} \phi_{\mathfrak{n}}(\mathbf{x})
\end{aligned}
$$

where

$$
\widetilde{\mathcal{G}}_{\mathfrak{n}, \mathfrak{M}}=\sum_{l=1}^{\infty}(-1)^{l}\left[\left\langle u_{\mathfrak{M}}\left(\mathcal{T}_{0}, \mathbf{x}\right), \phi_{\mathfrak{n}+2 l \mathfrak{M}}(\mathbf{x})\right\rangle+\left\langle u_{\mathfrak{M}}\left(\mathcal{T}_{0}, \mathbf{x}\right), \phi_{-\mathfrak{n}+2 l \mathfrak{M}}(\mathbf{x})\right\rangle\right] .
$$

We get that $\widetilde{\mathcal{G}}_{\mathfrak{n}, \mathfrak{M}}=0$ for $\mathfrak{n}>\mathfrak{M}$. Applying Lemma 2.1, we obtain

$$
f_{\mathfrak{M}}(\mathbf{x})=\sum_{\mathfrak{n}=1}^{\mathfrak{M}-1} \frac{\frac{\pi}{\mathfrak{M}} \sum_{\mathbf{k}=1}^{\mathfrak{M}} u_{\mathfrak{M}}\left(\mathcal{T}_{0}, \mathbf{x}_{\mathbf{k}}\right) \phi_{\mathfrak{n}}\left(\mathbf{x}_{\mathbf{k}}\right)}{\int_{0}^{\mathcal{T}_{0}} \mathbf{S}_{\mathfrak{n}}(\mathbf{t}-\mathfrak{s}, \gamma) \mathcal{Q}(\mathfrak{s}) d \mathfrak{s}} \phi_{\mathfrak{n}}(\mathbf{x})
$$

By the Parseval equality, we obtain

$$
\left\|f_{\mathfrak{M}}\right\|_{L^{2}(\Omega)}^{2}=\left[\sum_{\mathfrak{n}=1}^{\mathfrak{M}-1} \frac{\frac{\pi}{\mathfrak{M}} \sum_{\mathbf{k}=1}^{\mathfrak{M}} u_{\mathfrak{M}}\left(\mathcal{T}_{0}, \mathbf{x}_{\mathbf{k}}\right) \phi_{\mathfrak{n}}\left(\mathbf{x}_{\mathbf{k}}\right)}{\int_{0}^{\mathcal{T}_{0}} \mathbf{S}_{\mathfrak{n}}(\mathbf{t}-\mathfrak{s}, \gamma) \mathcal{Q}(\mathfrak{s}) d \mathfrak{s}}\right]^{2}
$$

Applying $\mathbb{E}\left(\varepsilon_{j} \varepsilon_{l}\right)=0(j \neq l), \mathbb{E}\left(\varepsilon_{j}\right)=0(j=1,2, \ldots, \mathfrak{n})$ with Lemma 2.1, we have

$$
\begin{aligned}
& \mathbb{E}\left\|f_{\mathfrak{M}}\right\|_{L^{2}(\Omega)}^{2}=\left[\sum_{\mathfrak{n}=1}^{\mathfrak{M}-1} \frac{\frac{\pi}{\mathfrak{M}} \sum_{\mathbf{k}=1}^{\mathfrak{M}} \frac{1}{\sqrt{\mathfrak{M}}} \mathbb{E} \varepsilon_{\mathbf{k}} \phi_{\mathfrak{n}}\left(\mathbf{x}_{\mathbf{k}}\right)}{\int_{0}^{\mathcal{T}_{0}} \mathbf{S}_{\mathfrak{n}}(\mathbf{t}-\mathfrak{s}, \gamma) \mathcal{Q}(\mathfrak{s}) d \mathfrak{s}}\right]^{2} \\
& =\sum_{\mathfrak{n}=1}^{\mathfrak{M}-1} \frac{\pi}{\mathfrak{M}^{2}}\left[\int_{0}^{\mathcal{T}_{0}} \mathbf{S}_{\mathfrak{n}}(\mathbf{t}-\mathfrak{s}, \gamma) \mathcal{Q}(\mathfrak{s}) d \mathfrak{s}\right]^{-2} .
\end{aligned}
$$

Using Lemma 3.3, we get

$$
\mathbb{E}\left\|f_{\mathfrak{M}}\right\|_{L^{2}(\Omega)}^{2} \geq \sum_{\mathfrak{n}=1}^{\mathfrak{M}-1} \frac{\pi}{\mathfrak{M}^{2}} \frac{\mathfrak{n}^{4} \gamma^{2}}{\|\mathcal{Q}\|_{\infty}^{2} \mathcal{B}_{2}^{2}(d, \gamma) \mathcal{T}_{0}^{2 \gamma}} \geq \frac{\pi}{\mathfrak{M}^{2}} \frac{(\mathfrak{M}-1)^{4} \gamma^{2}}{\|\mathcal{Q}\|_{\infty}^{2} \mathcal{B}_{2}^{2}(d, \gamma) \mathcal{T}_{0}^{2 \gamma}}
$$

Then

$$
\lim _{\mathfrak{M} \rightarrow \infty} \mathbb{E}\left\|f_{\mathfrak{M}}\right\|_{L^{2}(\Omega)}^{2}=0
$$

From the above argument, we can deduce that problem (1) is not well-posed. Hence, a regularization method is necessary.

\subsection{Regularization and convergence rate under a priori bounded condition}

We impose the following a priori bound on the exact solution $f(\mathbf{x})$. 
Theorem 4.1 Suppose $f \in \mathcal{H}^{\beta}(\Omega)$ and there exists $\mathbf{P}>0$ such that

$$
\|f\|_{\mathcal{H}^{\beta}(\Omega)} \leq \mathbf{P}, \quad \beta>0,
$$

then we have

$$
\|f\|_{L^{2}(\Omega)} \leq \frac{\mathbf{P}}{\frac{1}{\beta+1}\|\mathbf{h}\|_{L^{2}(\Omega)}^{\frac{\beta}{\beta+1}}}, \quad \beta>0 .
$$

Proof We have

$$
\begin{aligned}
\|f\|_{L^{2}(\Omega)}^{2} & =\left|\sum_{\mathfrak{n}=1}^{\infty} \frac{\left\langle\mathbf{h}(\mathbf{x}), \phi_{\mathfrak{n}}(\mathbf{x})\right\rangle}{\int_{0}^{\mathcal{T}_{0}} \mathbf{S}_{\mathfrak{n}}\left(\mathcal{T}_{0}-\mathfrak{s}, \gamma\right) \mathcal{Q}(\mathfrak{s}) d \mathfrak{s}}\right|^{2} \\
& =\sum_{\mathfrak{n}=1}^{\infty} \frac{\left|\left\langle\mathbf{h}(\mathbf{x}), \phi_{\mathfrak{n}}(\mathbf{x})\right\rangle\right|^{\frac{2}{\beta+1}}\left|\left\langle\mathbf{h}(\mathbf{x}), \phi_{\mathfrak{n}}(\mathbf{x})\right\rangle\right|^{\frac{2 \beta}{\beta+1}}}{\left|\int_{0}^{\mathcal{T}_{0}} \mathbf{S}_{\mathfrak{n}}\left(\mathcal{T}_{0}-\mathfrak{s}, \gamma\right) \mathcal{Q}(\mathfrak{s}) d \mathfrak{s}\right|^{2}} \\
& =\left.\sum_{\mathfrak{n}=1}^{\infty}\left[\frac{\left|\left\langle\mathbf{h}(\mathbf{x}), \phi_{\mathfrak{n}}(\mathbf{x})\right\rangle\right|^{2}}{\left|\int_{0}^{\mathcal{T}_{0}} \mathbf{S}_{\mathfrak{n}}\left(\mathcal{T}_{0}-\mathfrak{s}, \gamma\right) \mathcal{Q}(\mathfrak{s}) d \mathfrak{s}\right|^{2(\beta+1)}}\right]^{\frac{1}{\beta+1}}\left|\mathbf{h}(x), \phi_{\mathfrak{n}}(x)\right\rangle\right|^{\frac{2 \beta}{\beta+1}} \\
& =\sum_{\mathfrak{n}=1}^{\infty}\left[\frac{\left|\left\langle f(\mathbf{x}), \phi_{\mathfrak{n}}(\mathbf{x})\right\rangle\right|^{2}}{\left|\int_{0}^{\mathcal{T}_{0}} \mathbf{S}_{\mathfrak{n}}\left(\mathcal{T}_{0}-\mathfrak{s}, \gamma\right) \mathcal{Q}(\mathfrak{s}) d \mathfrak{s}\right|^{2 \beta}}\right]^{\frac{1}{\beta+1}}\left|\left\langle\mathbf{h}(\mathbf{x}), \phi_{\mathfrak{n}}(\mathbf{x})\right\rangle\right|^{\frac{2 \beta}{\beta+1}}
\end{aligned}
$$

Thanks to Lemma 3.3, we obtain

$$
\begin{aligned}
\|f\|_{L^{2}(\Omega)}^{2} & \left.\leq \sum_{\mathfrak{n}=1}^{\infty}\left[\frac{\lambda_{\mathfrak{n}}^{2 \beta}\left|\left\langle f(\mathbf{x}), \phi_{\mathfrak{n}}(\mathbf{x})\right\rangle\right|^{2}}{\mathcal{Q}_{0}^{2 \beta} \mathcal{B}_{1}^{2 \beta}\left(d, \mathcal{T}_{0}, \gamma\right)}\right]^{\frac{1}{\beta+1}}|| \mathbf{h}(\mathbf{x}), \phi_{\mathfrak{n}}(\mathbf{x})\right\rangle\left.\right|^{\frac{2 \beta}{\beta+1}} \\
& \leq \frac{\|f\|_{\mathcal{H}^{\beta}(\Omega)}^{\frac{2}{\beta+1}}\|\mathbf{h}\|_{L^{2}(\Omega)}^{\frac{2 \beta}{\beta+1}}}{\mathcal{Q}_{0}^{\frac{2 \beta}{\beta+1}} \mathcal{B}_{1}^{\frac{2 \beta}{\beta+1}}\left(d, \mathcal{T}_{0}, \gamma\right)} \leq \frac{\frac{2}{P^{\beta+1}}\|\mathbf{h}\|_{L^{2}(\Omega)}^{\frac{2 \beta}{\beta+1}}}{\mathcal{Q}_{0}^{\frac{2 \beta}{\beta+1}} \mathcal{B}_{1}^{\frac{2 \beta}{\beta+1}}\left(d, \mathcal{T}_{0}, \gamma\right)} .
\end{aligned}
$$

4.2.1 Error estimate in $L^{2}(\Omega)$

Theorem 4.2 Let $\epsilon>0$ and $\epsilon_{k} \sim N(0,1)$ with $\mathbf{k}=1, \ldots, \mathfrak{M}$ if the function $f(\mathbf{x})$ satisfies the prior bounded condition (4).

A regularized function $\widetilde{f}_{\mathfrak{M}, \mathcal{N}}$ is as follows:

$$
\tilde{f}_{\mathfrak{M}, \mathcal{N}}(\mathbf{x})=\sum_{\mathfrak{n}=1}^{\mathcal{N}} \frac{\frac{\pi}{\mathfrak{M}} \sum_{\mathbf{k}=1}^{\mathfrak{M}} \mathbf{G}\left(\mathbf{x}_{\mathbf{k}}\right) \phi_{\mathfrak{n}}\left(\mathbf{x}_{\mathbf{k}}\right)}{\int_{0}^{\mathcal{T}_{0}} \mathbf{S}_{\mathfrak{n}}\left(\mathcal{T}_{0}-\mathfrak{s}, \gamma\right) R(\mathfrak{s}) d \mathfrak{s}} \phi_{\mathfrak{n}}(\mathbf{x})
$$

where $\mathfrak{M}, \mathcal{N}$ are called regularization parameters. Then the error estimate between the exact source and its regularized source is as follows:

$$
\begin{aligned}
& \mathbb{E}\left\|\widetilde{f}_{\mathfrak{M}, \mathcal{N}}-f\right\|_{L^{2}(\Omega)}^{2} \\
& \quad \leq\left(\frac{\pi}{\mathfrak{M} \mathcal{Q}_{0}^{2} \mathcal{B}_{1}^{2}\left(d, \mathcal{T}_{0}, \gamma\right)} R_{\max }^{2}+\frac{\pi^{4}}{36} \frac{\mathcal{B}_{2}^{2}(d, \gamma)\|\mathcal{Q}\|_{\infty}^{2} \mathbf{P}^{\frac{2}{\beta+1}}\|\mathbf{h}\|_{L^{2}(\Omega)}^{\frac{2 \beta}{\beta+1}} \mathcal{T}_{0}^{2 \gamma}}{\gamma^{2} \mathfrak{M}^{4} \mathcal{Q}_{0}^{\frac{4 \beta+2}{\beta+1}} \mathcal{B}_{1}^{\frac{4 \beta+2}{\beta+1}}\left(d, \mathcal{T}_{0}, \gamma\right)}\right) N^{5} N^{-2 \beta} \mathbf{P}^{2}
\end{aligned}
$$


Let $\mathcal{N}:=\mathcal{N}_{\mathfrak{M}}$ such that $0<\mathcal{N}:=\mathcal{N}_{\mathfrak{M}}<\mathfrak{M}$ and

$$
\lim _{\mathfrak{M} \rightarrow+\infty} \frac{\mathcal{N}^{5}}{\mathfrak{M}}=0
$$

then

$$
\mathbb{E}\left\|\widetilde{f}_{\mathfrak{M}, \mathcal{N}}-f\right\|_{L^{2}(\Omega)}^{2} \text { is of order } \max \left(\frac{\mathcal{N}^{5}}{\mathfrak{M}}, \mathcal{N}^{-2 \beta}\right)
$$

Remark 4.1 By choosing $\mathcal{N}:=\mathfrak{M}^{\frac{1}{5+2 \beta}}$ and by (37), we can conclude that

$$
\mathbb{E}\left\|\tilde{f}_{\mathfrak{M}, \mathcal{N}}-f\right\|_{L^{2}(\Omega)}^{2} \text { is of order }\left(\frac{1}{\mathfrak{M}}\right)^{\frac{2 \beta}{5+2 \beta}}
$$

Proof of Theorem 4.2 First, we have the following estimate:

$$
\begin{aligned}
\widetilde{f}_{\mathfrak{M}, \mathcal{N}}(\mathbf{x})-f(\mathbf{x})= & \sum_{\mathfrak{n}=1}^{\mathcal{N}} \frac{\frac{\pi}{\mathfrak{M}} \sum_{\mathbf{k}=1}^{\mathfrak{M}} \sigma_{\mathbf{k}} \varepsilon_{\mathbf{k}} \phi_{\mathfrak{n}}\left(\mathbf{x}_{\mathbf{k}}\right)+\overline{\mathcal{G}}_{\mathfrak{n}, \mathfrak{M}}}{\int_{0}^{\mathcal{T}_{0}} \mathbf{S}_{\mathfrak{n}}\left(\mathcal{T}_{0}-\mathfrak{s}, \gamma\right) \mathcal{Q}(\mathfrak{s}) d \mathfrak{s}} \phi_{\mathfrak{n}}(\mathbf{x}) \\
& -\sum_{n=\mathcal{N}+1}^{\infty} \frac{\left\langle\mathbf{h}(\mathbf{x}), \phi_{\mathfrak{n}}(\mathbf{x})\right\rangle}{\int_{0}^{\mathcal{T}_{0}} \mathbf{S}_{\mathfrak{n}}\left(\mathcal{T}_{0}-\mathfrak{s}, \gamma\right) \mathcal{Q}(\mathfrak{s}) d \mathfrak{s}} \phi_{\mathfrak{n}}(\mathbf{x})
\end{aligned}
$$

This follows from the Parseval identity

$$
\begin{aligned}
\left\|\widetilde{f}_{\mathfrak{M}, \mathcal{N}}(\cdot)-f(\cdot)\right\|_{L^{2}(\Omega)}^{2}= & \sum_{\mathfrak{n}=1}^{\mathcal{N}}\left[\frac{\frac{\pi}{\mathfrak{M}} \sum_{\mathbf{k}=1}^{\mathfrak{M}} \sigma_{\mathbf{k}} \varepsilon_{\mathbf{k}} \phi_{\mathfrak{n}}\left(\mathbf{x}_{\mathbf{k}}\right)-\overline{\mathcal{G}}_{\mathfrak{n}, \mathfrak{M}}}{\int_{0}^{\mathcal{T}_{0}} \mathbf{S}_{\mathfrak{n}}\left(\mathcal{T}_{0}-\mathfrak{s}, \gamma\right) \mathcal{Q}(\mathfrak{s}) d \mathfrak{s}}\right]^{2} \\
& +\sum_{\mathfrak{n}=\mathcal{N}+1}^{\infty}\left[\frac{\left\langle\mathbf{h}(\mathbf{x}), \phi_{\mathfrak{n}}(\mathbf{x})\right\rangle}{\int_{0}^{\mathcal{T}_{0}} \mathbf{S}_{\mathfrak{n}}\left(\mathcal{T}_{0}-\mathfrak{s}, \gamma\right) \mathcal{Q}(\mathfrak{s}) d \mathfrak{s}}\right]^{2}
\end{aligned}
$$

The fact that $\mathbb{E}\left(\varepsilon_{j} \varepsilon_{l}\right)=0(l \neq j)$, and $\mathbb{E}\left(\varepsilon_{j}\right)=0(j=\overline{1, \mathfrak{n}})$. So we can deduce that

$$
\begin{aligned}
\mathbb{E} & \left\|\tilde{f}_{\mathfrak{M}, \mathcal{N}}(\cdot)-f(\cdot)\right\|_{L^{2}(\Omega)}^{2} \\
& =\sum_{\mathfrak{n}=\mathcal{N}+1}^{\infty}\left[\frac{\left\langle\mathbf{h}(\mathbf{x}), \phi_{\mathfrak{n}}(\mathbf{x})\right\rangle}{\int_{0}^{\mathcal{T}_{0}} \mathbf{S}_{\mathfrak{n}}\left(\mathcal{T}_{0}-\mathfrak{s}, \gamma\right) \mathcal{Q}(\mathfrak{s}) d \mathfrak{s}}\right]^{2}+\sum_{\mathfrak{n}=1}^{\mathcal{N}} \frac{\frac{\pi^{2}}{\mathfrak{M}^{2}} \sum_{\mathbf{k}=1}^{\mathfrak{M}} \sigma_{\mathbf{k}}^{2} \mathbb{E} \varepsilon_{\mathbf{k}}^{2}+\overline{\mathcal{G}}_{\mathfrak{n}, \mathfrak{M}}^{2}}{\left[\int_{0}^{\mathcal{T}_{0}} \mathbf{S}_{\mathfrak{n}}\left(\mathcal{T}_{0}-\mathfrak{s}, \gamma\right) \mathcal{Q}(\mathfrak{s}) d \mathfrak{s}\right]^{2}} \\
& =\mathbb{I}_{1}+\mathbb{I}_{2} .
\end{aligned}
$$

We have

$$
\mathbb{I}_{1}=\sum_{\mathfrak{n}=\mathcal{N}+1}^{\infty}\left[\frac{\left\langle\mathbf{h}(\mathbf{x}), \phi_{\mathfrak{n}}(\mathbf{x})\right\rangle}{\int_{0}^{\mathcal{T}_{0}} \mathbf{S}_{\mathfrak{n}}\left(\mathcal{T}_{0}-\mathfrak{s}, \gamma\right) \mathcal{Q}(\mathfrak{s}) d \mathfrak{s}}\right]^{2}
$$

By equation (25), we know that, for $\mathfrak{n} \geq 1$,

$$
\left\langle f(\mathbf{x}), \phi_{\mathfrak{n}}(\mathbf{x})\right\rangle=\frac{\left\langle\mathbf{h}(\mathbf{x}), \phi_{\mathfrak{n}}(\mathbf{x})\right\rangle}{\int_{0}^{\mathcal{T}_{0}} \mathbf{S}_{\mathfrak{n}}\left(\mathcal{T}_{0}-\mathfrak{s}, \gamma\right) \mathcal{Q}(\mathfrak{s}) d \mathfrak{s}} .
$$


Using the last two equations, we get

$$
\mathbb{I}_{1}=\sum_{\mathfrak{n}=\mathcal{N}+1}^{\infty}\left[\left\langle f(\mathbf{x}), \phi_{\mathfrak{n}}(\mathbf{x})\right\rangle\right]^{2}
$$

By $1=\mathfrak{n}^{-2 \beta} \mathfrak{n}^{2 \beta}$, we can rewrite $\mathbb{I}_{1}$ as follows:

$$
\mathbb{I}_{1}=\sum_{\mathfrak{n}=\mathcal{N}+1}^{\infty} \mathfrak{n}^{-2 \beta} \mathfrak{n}^{2 \beta}\left|\left\langle f(\mathbf{x}), \phi_{\mathfrak{n}}(\mathbf{x})\right\rangle\right|^{2}
$$

In the last series (42), since $\mathfrak{n} \geq \mathcal{N}+1>\mathcal{N}$, we get $\mathfrak{n}^{-2 \beta} \leq \mathcal{N}^{-2 \beta}$. Using the last two observations, we obtain

$$
\mathbb{I}_{1} \leq \sum_{\mathfrak{n}=\mathcal{N}+1}^{\infty} \mathcal{N}^{-2 \beta} \mathfrak{n}^{2 \beta}\left|\left\langle f(\mathbf{x}), \phi_{\mathfrak{n}}(\mathbf{x})\right\rangle\right|^{2}=\mathcal{N}^{-2 \beta} \sum_{\mathfrak{n}=\mathcal{N}+1}^{\infty} \mathfrak{n}^{2 \beta}\left|\left\langle f(\mathbf{x}), \phi_{\mathfrak{n}}(\mathbf{x})\right\rangle\right|^{2}=\mathcal{N}^{-2 \beta} \widehat{\mathbb{I}}_{1}
$$

We shall begin with showing that

$$
\widehat{\mathbb{I}}_{1}=\sum_{\mathfrak{n}=\mathcal{N}+1}^{\infty} \mathfrak{n}^{2 \beta}\left|\left\langle f(\mathbf{x}), \phi_{\mathfrak{n}}(\mathbf{x})\right\rangle\right|^{2} \leq \sum_{\mathfrak{n}=1}^{\infty} \mathfrak{n}^{2 \beta}\left|\left\langle f(\mathbf{x}), \phi_{\mathfrak{n}}(\mathbf{x})\right\rangle\right|^{2}=\|f\|_{\mathcal{H}^{\beta}(\Omega)}^{2} .
$$

Using (43) and (44), we get

$$
\mathbb{I}_{1} \leq \mathcal{N}^{-2 \beta}\|f\|_{\mathcal{H}(\Omega)}^{2} \leq \mathcal{N}^{-2 \beta} \mathbf{P}^{2}
$$

Recall the definition of $\mathbb{I}_{2}$ in equation (40)

$$
\begin{aligned}
\mathbb{I}_{2} & =\sum_{\mathfrak{n}=1}^{N} \frac{\frac{\pi^{2}}{\mathfrak{M}^{2}} \sum_{\mathbf{k}=1}^{\mathfrak{M}} \sigma_{\mathbf{k}}^{2} \mathbb{E} \varepsilon_{\mathbf{k}}^{2}+\overline{\mathcal{G}}_{\mathfrak{n}, \mathfrak{M}}^{2}}{\left[\int_{0}^{\mathcal{T}_{0}} \mathbf{S}_{\mathfrak{n}}\left(\mathcal{T}_{0}-\mathfrak{s}, \gamma\right) \mathcal{Q}(\mathfrak{s}) d \mathfrak{s}\right]^{2}} \\
& =\left(\frac{\pi^{2}}{\mathfrak{M}^{2}} \sum_{\mathbf{k}=1}^{\mathfrak{M}} \sigma_{\mathbf{k}}^{2} \mathbb{E} \varepsilon_{\mathbf{k}}^{2}+\overline{\mathcal{G}}_{\mathfrak{n}, \mathfrak{M}}^{2}\right) \sum_{\mathfrak{n}=1}^{\mathcal{N}}\left[\int_{0}^{\mathcal{T}_{0}} \mathbf{S}_{\mathfrak{n}}\left(\mathcal{T}_{0}-\mathfrak{s}, \gamma\right) \mathcal{Q}(\mathfrak{s}) d \mathfrak{s}\right]^{-2} .
\end{aligned}
$$

We invoke Lemma 3.3 to deduce that

$$
\begin{aligned}
\left|\left\langle\mathbf{h}(\mathbf{x}), \phi_{\mathfrak{n}}(\mathbf{x})\right\rangle\right| & =\left[\int_{0}^{\mathcal{T}_{0}} \mathbf{S}_{\mathfrak{n}}\left(\mathcal{T}_{0}-\mathfrak{s}, \gamma\right) \mathcal{Q}(\mathfrak{s}) d \mathfrak{s}\right]\left|\left\langle f(\mathbf{x}), \phi_{\mathfrak{n}}(\mathbf{x})\right\rangle\right| \\
& \leq\|\mathcal{Q}\|_{\infty} \frac{\mathcal{B}_{2}(d, \gamma)}{\mathfrak{n}^{2}} \frac{\mathcal{T}_{0}^{\gamma}}{\gamma}\|f\|_{L^{2}(\Omega)},
\end{aligned}
$$

where $\sum_{l=1}^{\infty} \frac{1}{l^{2}}=\frac{\pi^{2}}{6}$. We can now combine the results of Lemma 2.2 and equation (47) to obtain

$$
\begin{aligned}
& \overline{\mathcal{G}}_{\mathfrak{n}, \mathfrak{M}} \leq \sum_{l=1}^{\infty}\left[\left\langle\mathbf{h}(\mathbf{x}), \phi_{\mathfrak{n}+2 l \mathfrak{M}}(\mathbf{x})\right\rangle+\left\langle\mathbf{h}(\mathbf{x}), \phi_{-\mathfrak{n}+2 l \mathfrak{M}}(\mathbf{x})\right\rangle\right] \\
& \leq \frac{\mathcal{B}_{2}(d, \gamma) \mathcal{T}_{0}^{\gamma}\|\mathcal{Q}\|_{\infty}\|f\|_{L^{2}(\Omega)}}{\gamma}\left[\sum_{l=1}^{\infty} \frac{1}{(\mathfrak{n}+2 l \mathfrak{M})^{2}}+\sum_{l=1}^{\infty} \frac{1}{(-\mathfrak{n}+2 l \mathfrak{M})^{2}}\right]
\end{aligned}
$$




$$
\begin{aligned}
& \leq \frac{\pi^{2}}{6} \frac{\mathcal{B}_{2}(d, \gamma) \mathcal{T}_{0}^{\gamma}\|\mathcal{Q}\|_{\infty}\|f\|_{L^{2}(\Omega)}}{\gamma \mathfrak{M}^{2}} \\
& \leq \frac{\pi^{2}}{6} \frac{\mathcal{B}_{2}(d, \gamma)\|\mathcal{Q}\|_{\infty} \mathbf{P}^{\frac{1}{\beta+1}}\|\mathbf{h}\|_{L^{2}(\Omega)}^{\frac{\beta}{\beta+1}} \mathcal{T}_{0}^{\gamma}}{\gamma \mathfrak{M}^{2} \mathcal{Q}_{0}^{\frac{\beta}{\beta+1}} \mathcal{B}_{1}^{\frac{\beta}{\beta+1}}\left(d, \mathcal{T}_{0}, \gamma\right)}
\end{aligned}
$$

Since $\sigma_{k}<R_{\max }$, we estimate $\mathbb{I}_{2}$ as follows:

$$
\begin{aligned}
\mathbb{I}_{2} \leq & \left(\frac{\pi}{\mathfrak{M}} R_{\max }^{2}+\frac{\pi^{4}}{36} \frac{\mathcal{B}_{2}^{2}(d, \gamma)\|\mathcal{Q}\|_{\infty}^{2} \mathbf{P}^{\frac{2}{\beta+1}}\|\mathbf{h}\|_{L^{2}(\Omega)}^{\frac{2 \beta}{\beta+1}} \mathcal{T}_{0}^{2 \gamma}}{\gamma^{2} \mathfrak{M}^{4} \mathcal{Q}_{0}^{\frac{2 \beta}{\beta+1}} \mathcal{B}_{1}^{\frac{2 \beta}{\beta+1}}\left(d, \mathcal{T}_{0}, \gamma\right)}\right) \\
& \times \sum_{\mathfrak{n}=1}^{\mathcal{N}}\left[\int_{0}^{\mathcal{T}_{0}} \mathbf{S}_{\mathfrak{n}}\left(\mathcal{T}_{0}-\mathfrak{s}, \gamma\right) \mathcal{Q}(\mathfrak{s}) d \mathfrak{s}\right]^{-2} \\
\leq & \left(\frac{\pi}{\mathfrak{M}} R_{\max }^{2}+\frac{\pi^{4}}{36} \frac{\mathcal{B}_{2}^{2}(d, \gamma)\|\mathcal{Q}\|_{\infty}^{2} \mathbf{P}^{\frac{2}{\beta+1}}\|\mathbf{h}\|_{L^{2}(\Omega)}^{\frac{2 \beta}{\beta+1}} \mathcal{T}_{0}^{2 \gamma}}{\gamma^{2} \mathfrak{M}^{4} \mathcal{Q}_{0}^{\frac{2 \beta}{\beta+1}} \mathcal{B}_{1}^{\frac{2 \beta}{\beta+1}}\left(d, \mathcal{T}_{0}, \gamma\right)} \sum_{\mathfrak{n}=1}^{\mathcal{N}} \frac{\mathfrak{n}^{4}}{\mathcal{Q}_{0}^{2} \mathcal{B}_{1}^{2}\left(d, \mathcal{T}_{0}, \gamma\right)}\right. \\
\leq & \left(\frac{\pi}{\mathfrak{M} \mathcal{Q}_{0}^{2} \mathcal{B}_{1}^{2}\left(d, \mathcal{T}_{0}, \gamma\right)} R_{\max }^{2}+\frac{\pi^{4}}{36} \frac{\mathcal{B}_{2}^{2}(d, \gamma)\|\mathcal{Q}\|_{\infty}^{2} \mathbf{P}^{\frac{2}{\beta+1}}\|\mathbf{h}\|_{L^{2}(\Omega)}^{\frac{2 \beta}{\beta+1}} \mathcal{T}_{0}^{2 \gamma}}{\gamma^{2} \mathfrak{M}^{4} \mathcal{Q}_{0}^{\frac{4 \beta+2}{\beta+1}}} \mathcal{B}_{1}^{\frac{4 \beta+1}{\beta+1}}\left(d, \mathcal{T}_{0}, \gamma\right)\right.
\end{aligned}
$$

Combining equation (45) with equation (50), we obtain

$$
\begin{aligned}
& \mathbb{E}\left\|\widetilde{f}_{\mathfrak{M}, N}-f\right\|_{L^{2}(\Omega)}^{2} \\
& \quad \leq\left(\frac{\pi}{\mathfrak{M} \mathcal{Q}_{0}^{2} \mathcal{B}_{1}^{2}\left(d, \mathcal{T}_{0}, \gamma\right)} R_{\max }^{2}+\frac{\pi^{4}}{36} \frac{\mathcal{B}_{2}^{2}(d, \gamma)\|\mathcal{Q}\|_{\infty}^{2} \mathbf{P}^{\frac{2}{\beta+1}}\|\mathbf{h}\|_{L^{2}(\Omega)}^{\frac{2 \beta}{\beta+1}} \mathcal{T}_{0}^{2 \gamma}}{\gamma^{2} \mathfrak{M}^{4} \mathcal{Q}_{0}^{\frac{4 \beta+2}{\beta+1}} \mathcal{B}_{1}^{\frac{4 \beta+2}{\beta+1}}\left(d, \mathcal{T}_{0}, \gamma\right)}\right) \mathcal{N}^{5}+\mathcal{N}^{-2 \beta} \mathbf{P}^{2}
\end{aligned}
$$

It is shown that our main results are stated and proved.

\subsubsection{Error estimate in $\mathcal{H}^{\beta}$}

Now, we give error estimate between the source and the regularized source in higher Sobolev spaces. For any $\beta>0$, the convergence rate in $\mathcal{H}^{\beta}$ norm is as follows.

Theorem 4.3 Assume that $f(\mathbf{x}) \in \mathcal{H}^{\beta+\mathfrak{m}}$ for any $\mathfrak{m}>0$. Then

$$
\mathbb{E}\left\|\widetilde{f}_{\mathfrak{M}, \mathcal{N}}-f\right\|_{\mathcal{H}^{\beta}(\Omega)}^{2} \text { is of order } \max \left(\frac{\mathcal{N}^{5+2 \beta}}{\mathfrak{M}}, \mathcal{N}^{-2 m}\right)
$$

Proof We have

$$
\left\|\widetilde{f}_{\mathfrak{M}, \mathcal{N}}-f\right\|_{\mathcal{H}^{\beta}(\Omega)}^{2}=\sum_{\mathfrak{n}=1}^{\infty} \lambda_{\mathfrak{n}}^{\beta}\left|\left\langle\widetilde{f}_{\mathfrak{M}, \mathcal{N}}-f, \varphi_{\mathfrak{n}}(\mathbf{x})\right\rangle\right|^{2}=\left.\sum_{\mathfrak{n}=1}^{\infty} \mathfrak{n}^{2 \beta}\left|\widetilde{f}_{\mathfrak{M}, \mathcal{N}}-f, \varphi_{\mathfrak{n}}(\mathbf{x})\right\rangle\right|^{2}
$$


Take the expectation to both sides and use Theorem 4.2. The fact that $\mathbb{E}\left(\varepsilon_{j} \varepsilon_{l}\right)=0(j \neq l)$, and $\mathbb{E}\left(\varepsilon_{j}\right)=0(j=\overline{1, \mathfrak{n}})$. Now we can deduce that

$$
\begin{aligned}
\mathbb{E}\left\|\widetilde{f}_{\mathfrak{M}, \mathcal{N}}-f\right\|_{\mathcal{H}^{\beta}(\Omega)}^{2}= & \sum_{\mathfrak{n}=\mathcal{N}+1}^{\infty} \mathfrak{n}^{2 \beta}\left[\frac{\left\langle\mathbf{h}(\mathbf{x}), \phi_{\mathfrak{n}}(\mathbf{x})\right\rangle}{\int_{0}^{\mathcal{T}_{0}} \mathbf{S}_{\mathfrak{n}}\left(\mathcal{T}_{0}-\mathfrak{s}, \gamma\right) \mathcal{Q}(\mathfrak{s}) d \mathfrak{s}}\right]^{2} \\
& +\sum_{\mathfrak{n}=1}^{\mathcal{N}} \mathfrak{n}^{2 \beta} \frac{\frac{\pi^{2}}{\mathfrak{M}^{2}} \sum_{\mathbf{k}=1}^{\mathfrak{M}} \sigma_{\mathbf{k}}^{2} \mathbb{E} \varepsilon_{\mathbf{k}}^{2}+\overline{\mathcal{G}}_{\mathfrak{n}, \mathfrak{M}}^{2}}{\left[\int_{0}^{\mathcal{T}_{0}} \mathbf{S}_{\mathfrak{n}}\left(\mathcal{T}_{0}-\mathfrak{s}, \gamma\right) \mathcal{Q}(\mathfrak{s}) d \mathfrak{s}\right]^{2}} \\
= & \widetilde{\mathbb{J}}_{1}+\widetilde{\mathbb{J}}_{2} .
\end{aligned}
$$

First, using equation (25), we get

$$
\widetilde{\mathbb{J}}_{1}=\sum_{\mathfrak{n}=\mathcal{N}+1}^{\infty} \mathfrak{n}^{-2 \mathfrak{m}} \mathfrak{n}^{2 \beta+2 \mathfrak{m}} \mid\left\langle f(\mathbf{x}),\left.\phi_{\mathfrak{n}}(\mathbf{x})\right|^{2} \leq \mathcal{N}^{-2 \mathfrak{m}}\|f\|_{\mathcal{H}^{\beta+\mathfrak{m}}(\Omega)}^{2}\right.
$$

We can now proceed analogously to the proof of Theorem 4.2, we continue to estimate the error $\widetilde{\mathbb{I}}_{2}$ :

$$
\begin{aligned}
\widetilde{\mathbb{J}}_{2} & =\left(\frac{\pi^{2}}{\mathfrak{M}^{2}} \sum_{\mathbf{k}=1}^{\mathfrak{M}} \sigma_{\mathbf{k}}^{2} \mathbb{E} \varepsilon_{\mathbf{k}}^{2}+\overline{\mathcal{G}}_{\mathfrak{n}, \mathfrak{M}}^{2}\right) \sum_{\mathfrak{n}=1}^{\mathcal{N}} \mathfrak{n}^{2 \beta}\left[\int_{0}^{\mathcal{T}_{0}} \mathbf{S}_{\mathfrak{n}}\left(\mathcal{T}_{0}-\mathfrak{s}, \gamma\right) \mathcal{Q}(\mathfrak{s}) d \mathfrak{s}\right]^{-2} \\
& \leq\left(\frac{\pi}{\mathfrak{M}^{2}} R_{\max }^{2}+\frac{\pi^{4}}{36} \frac{\mathcal{B}_{2}^{2}(d, \gamma)\|\mathcal{Q}\|_{\infty}^{2} \mathbf{P}^{\frac{2}{\beta+1}}\|\mathbf{h}\|_{L^{2}(\Omega)}^{\frac{2 \beta}{\beta+1}} \mathcal{T}_{0}^{2 \gamma}}{\gamma^{2} \mathfrak{M}^{4} \mathcal{Q}_{0}^{\frac{2 \beta}{\beta+1}} \mathcal{B}_{1}^{\frac{2 \beta}{\beta+1}}\left(d, \mathcal{T}_{0}, \gamma\right)}\right) \sum_{\mathfrak{n}=1}^{\mathcal{N}} \frac{\mathfrak{n}_{0}^{4+2 \beta}}{\mathcal{Q}_{0}^{2} \mathcal{B}_{1}^{2}\left(d, \mathcal{T}_{0}, \gamma\right)} \\
& \leq\left(\frac{\pi}{\mathfrak{M} \mathcal{Q}_{0}^{2} \mathcal{B}_{1}^{2}\left(d, \mathcal{T}_{0}, \gamma\right)} R_{\max }^{2}+\frac{\pi^{4}}{36} \frac{\mathcal{B}_{2}^{2}(d, \gamma)\|\mathcal{Q}\|_{\infty}^{2} \mathbf{P}^{\frac{2}{\beta+1}}\|\mathbf{h}\|_{L^{2}(\Omega)}^{\frac{2 \beta}{\beta+1}} \mathcal{T}_{0}^{2 \gamma}}{\gamma^{2} \mathfrak{M}^{4} \mathcal{Q}_{0}^{\frac{4 \beta+2}{\beta+1}} \mathcal{B}_{1}^{\frac{4 \beta+1}{\beta+1}}\left(d, \mathcal{T}_{0}, \gamma\right)}\right) \mathcal{N}^{5+2 \beta} .
\end{aligned}
$$

Combining (54) and (55), we obtain (52) immediately.

\section{Acknowledgements}

The authors would like to thank the editor and reviewers in helping to improve the manuscript.

Funding

Not applicable.

\section{Availability of data and materials}

Not applicable.

\section{Competing interests}

The authors declare that they have no competing interests.

\section{Authors' contributions}

The authors declare that the study was realized in collaboration with the same responsibility. All authors contributed equally to the writing of this paper. All authors read and approved the final manuscript.

\section{Author details}

${ }^{1}$ Faculty of Natural Sciences, Thu Dau Mot University, Thu Dau Mot City, Vietnam. ${ }^{2}$ Department of Mathematics, Cankaya University, Ankara, Turkey. ${ }^{3}$ Department of Medical Research, China Medical University Hospital, China Medical University, Taichung, Taiwan. ${ }^{4}$ Institute of Space Sciences, Magurele-Bucharest, Romania. ${ }^{5}$ Institute of Fundamental and Applied Sciences, Duy Tan University, Ho Chi Minh City, Vietnam. ${ }^{6}$ Applied Analysis Research Group, Faculty of Mathematics and Statistics, Ton Duc Thang University, Ho Chi Minh City, Vietnam. 


\section{Publisher's Note}

Springer Nature remains neutral with regard to jurisdictional claims in published maps and institutional affiliations.

Received: 3 October 2019 Accepted: 28 November 2019 Published online: 05 December 2019

\section{References}

1. Baleanu, D., Scalas, E., Diethelm, K., Trujillo, J.J.: Fractional Calculus: Models and Numerical Methods. World Scientific, Singapore (2012)

2. Caputo, M.: Linear models of dissipation whose $Q$ is almost frequency independent, part II. Geophys. J. R. Astron. Soc. 13(5), 529-539 (1967)

3. Kilbas, A.A., Srivastava, H.M., Trujillo, J.J.: Theory and Application of Fractional Differential Equations. North-Holland Mathematics Studies, vol. 204. Elsevier, Amsterdam (2006)

4. Fernandez, A., Ozarslan, A.M., Baleanu, D.: On fractional calculus with general analytic kernels. Appl. Math. Comput. $354,248-265$ (2019)

5. Kilbas, A.A., Srivastava, H.M., Trujillo, J.J.: Theory and Applications of Fractional Differential Equations. Elsevier, Amsterdam (2006)

6. Zhou, Y., Shangerganesh, L., Manimaran, J., Debbouche, A.: A class of time-fractional reaction-diffusion equation with nonlocal boundary condition. Math. Methods Appl. Sci. 41, 2987-2999 (2018)

7. Zhou, Y., Peng, L., Huang, Y.Q.: Duhamel's formula for time-fractional Schrödinger equations. Math. Methods Appl. Sci. 41, 8345-8349 (2018)

8. Zhou, Y., Peng, L., Huang, Y.Q.: Existence and Hölder continuity of solutions for time-fractional Navier-Stokes equations. Math. Methods Appl. Sci. 41, 7830-7838 (2018)

9. Podlubny, I.: Fractional Differential Equations. Mathematics in Science and Engineering, vol. 198. Academic Press, San Diego (1990)

10. Shen, F., Tan, W., Zhao, Y., Masuoka, T.: The Rayleigh-Stokes problem for a heated generalized second grade fluid with fractional derivative model. Nonlinear Anal., Real World Appl. 7(5), 1072-1080 (2006)

11. Triet, N.A., Hoan, L.V.C., Luc, N.H., Tuan, N.H., Thinh, N.V.: Identification of source term for the Rayleigh-Stokes problem with Gaussian random noise. Math. Methods Appl. Sci. 41(14), 5593-5601 (2018)

12. Dehghan, M.: A computational study of the one-dimensional parabolic equation subject to nonclassical boundary specifications. Numer. Methods Partial Differ. Equ. 22(1), 220-257 (2006)

13. Dehghan, M.: The one-dimensional heat equation subject to a boundary integral specification. Chaos Solitons Fractals 32(2), 661-675 (2007)

14. Dehghan, M., Abbaszadeh, M.: A finite element method for the numerical solution of Rayleigh-Stokes problem for a heated generalized second grade fluid with fractional derivatives. Eng. Comput. 33, 587-605 (2017)

15. Mehrdad, L., Dehghan, M.: The use of Chebyshev cardinal functions for the solution of a partial differential equation with an unknown time-dependent coefficient subject to an extra measurement. J. Comput. Appl. Math. 235(3), 669-678 (2010)

16. Zaky, A.M.: An improved tau method for the multi-dimensional fractional Rayleigh-Stokes problem for a heated generalized second grade fluid. Comput. Math. Appl. 75(7), 2243-2258 (2018)

17. Bazhlekova, E., Jin, B., Lazarov, R., Zhou, Z:: An analysis of the Rayleigh-Stokes problem for a generalized second-grade fluid. Numer. Math. 131, 1-31 (2015)

\section{Submit your manuscript to a SpringerOpen ${ }^{\circ}$ journal and benefit from:}

- Convenient online submission

- Rigorous peer review

- Open access: articles freely available online

- High visibility within the field

- Retaining the copyright to your article

Submit your next manuscript at $\gg$ springeropen.com 